\section{Fusing ER}

The endoplasmic reticulum (ER) forms a tubular network in the cell and homotypic membrane fusion is required for its biogenesis and maintenance. ER fusion involves GTP (guanosine triphosphate) hydrolysis and Orso et al. now report that the dynamin-like GTPase Atlastin mediates homotypic ER fusion (Nature doi: 10.1038/nature08280).

Human Atlastins had previously been implicated in Golgi and ER morphogenesis. Orso et al., confirm that fly Atlastin is an ER-localized protein; its loss leads to ER fragmentation whereas overexpression causes ER expansion with no effect on secretory traffic. Like human Atlastins, fly Atlastin homo-oligomerizes. Furthermore, Atlastin self-associates when present on different vesicles, suggesting a tethering function. By demonstrating that Atlastin was sufficient to promote fusion of liposomes in vitro, the authors provide crucial evidence for a direct role for Atlastin in membrane fusion. A mutant lacking GTPase activity was unable to mediate fusion in vitro or to homo-oligomerize in cells, and it failed to rescue the ER fragmentation defect of Atlastin mutant flies. Two other papers also describe a role for mammalian Atlastin in ER morphology (J. Neurochem. 110, 1607-1616; 2009 and Cell 138, 549-561; 2009).

Mutations in human Atlastin are associated with hereditary spastic paraplegia and whether these mutations perturb Atlastinmediated ER membrane fusion is an open question.

\section{iPS mice}

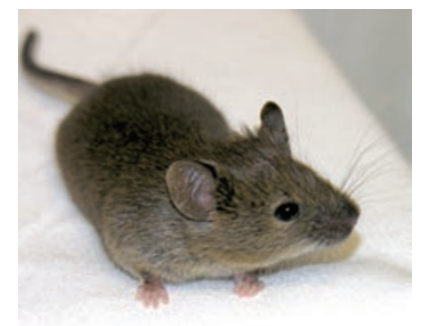

From Boland et al., Nature doi: 10.1038/ nature08310; 2009

Induced pluripotent stem (iPS) cell lines can generate chimaeras and are competent for germline transmission but, so far, they have been unable to pass the most stringent test for pluripotency, the generation of adult mice through tetraploid blastocyst complementation, as proven for embryonic stem (ES) cells and somatic nuclear transfer ES (SCNT-ES) cells. Three independent studies now report murine iPS cell lines that can support full-term development (Kang et al., Cell Stem Cell 5, 135-138; 2009) and the generation of reproductively competent adult mice (Zhao et al., Nature doi: 10.1038/nature08267; 2009 and Boland et al., Nature doi: 10.1038/nature08310; 2009), when injected into host tetraploid blastocysts. Any viable mice obtained through this method must be derived primarily from the injected diploid iPS cells, which generate the embryo proper, whereas the tetraploid blastocyst contributes to the extraembryonic lineages.

In the three studies, iPS cells were obtained by expressing the reprogramming factors Oct4, Sox2, Klf4 and c-Myc in mouse embryonic

\title{
mRNA decay turns on apoptosis
}

As part of the adaptative response to endoplasmic reticulum (ER) stress, the ER transmembrane kinase and endoribonuclease IRE1 $\alpha$ cleaves the mRNA of the transcription factor XBP1. The resulting XBP1 variant upregulates expression of proteins that enhance ER folding capacity. IRE1 a was previously shown to degrade a range of mRNAs in Drosophila. Papa and colleagues (Cell 138, 562-575; 2009) and Weissman and colleagues (J. Cell Biol. 186, 323-331; 2009) have found that in mammalian cells, the mode of activation of IRE 1a changes its specificity from XBP1 to a more general degradation of ER-localized mRNAs.

Both studies suggest that high levels of ER stress cause IRE1 $\alpha$ to trigger decay of mRNA encoding ER cargo, including chaperones. This effect requires the activation of its endoribonuclease activity. Based on recent structural data, chronic ER stress could induce IRE1 a to form higher order oligomers, which may display a higher promiscuity towards mRNA. Papa and colleagues further demonstrate that this IRE1 $\alpha$-mediated mRNA decay impairs cell function and induces apoptosis. The divergent outcomes of IRE1 $\alpha$ activity on cells provide one explanation for how the unfolded protein response switches to apoptosis when cell homeostasis is not restored.

fibroblasts and selecting iPS clones on the basis of pluripotency marker expression, ES-like morphology, epigenetic profile, normal karyotype, teratoma and chimaera formation ability. Live-born viable iPS pups had no obvious morphological abnormalities, but the overall efficiency of the process was low: 2 live pups out of 187 injected blastocysts in Kang et al., 27 out of 848 in Zhao et al. and 29 out of 730 in Boland et al., of which a significant fraction survived into adulthood.

These findings unequivocally demonstrate that iPS cell lines can generate all the cell types in an organism. Further investigation should show which attributes are critical for the higher developmental capacity of the successful iPS lines, and whether full pluripotency can also be achieved by reprogramming adult fibroblasts.

SG

\section{Par3 positions the \\ centrosome}

During fibroblast migration, the centrosome becomes oriented towards the leading edge; dynein keeps the centrosome in place while the nucleus moves rearward. Gundersen and colleagues (Curr. Biol. 19, 1065-1074; 2009) now report that dynein is anchored by the polarity protein Par3, which regulates microtubule dynamics at cell-cell contacts.

The authors find that Par3 functions as a cortical anchor for dynein, associating specifically with its LIC2 (light intermediate chain 2) subunit. Loss of either Par3 or LIC2 inhibited the centrosome-centring pathway and the authors show that LIC2 mediates Par3 localization to cell-cell contacts, which coincide with microtubule ends. Both Par3 and LIC2 were necessary for increased microtubule pausing seen at cellcell contacts in response to lysophosphatidic acid; conversely, overexpression of the Par3 fragment that binds LIC2 disrupted both microtubule pausing and centrosome orientation.

The authors propose that Par3 tethers dynein, which keeps the centrosome in place through altered microtubule dynamics. It is possible, therefore, that this model may apply to other situations where dynein affects microtubule dynamics.

AS

Written by Silvia Grisendi, Nathalie Le Bot Alison Schuldt and Sowmya Swaminathan 